\title{
The leads are still the weakest link
}

\author{
Jordan M. Prutkin, Arun R. Sridhar \\ Division of Cardiology, Section of Electrophysiology, University of Washington, Seattle, WA, Unites States
}

Article Dębski et al., see p. 1224

"The weakest link in a chain is the strongest because it can break it"

(Stanisław Lec [1])

Since the invention of the first wearable pacemaker in 1958, the field of cardiac pacing has made a remarkable progress [2]. Much of the effort has gone into the improvement of generator longevity, size, and features. Lead development, on the other hand, has not been as much of a focus. Perhaps this is because operators and patients just expect leads to work. Leads form the vital connection through the vascular system to the heart and are subjected to immense amount of friction, inflammation, and fibrosis and the perpetual motion of the heart. Lead failures are much more common compared to generator and battery failures and pose management quandaries in terms of whether to abandon or extract them when implanting fresh leads [3].

The study by Dębski et al. [4] is a timely article which discusses the issue of lead longevity. The authors should be congratulated for their work. With data from 3771 patients over 30 years and a combined follow-up period of 24,432 patient-years, this study is one of the largest single-centre analyses of pacemaker lead-related complications. The authors studied three different lead dysfunctions: 1) lead dislodgement, 2) cardiac perforation, and 3) lead failure. The overall lead dysfunction rate was $5.5 \%$ of all leads, with the majority being lead failure $(4.2 \%)$ followed by lead dislodgement $(1.2 \%)$, and, rarely, cardiac perforation $(0.1 \%)$

\section{LEAD DISLODGEMENT}

Lead dislodgement occurred in $2.4 \%$ of the patients. The only independent predictor of dislodgement was implant in the atrial lead position. Other studies have similarly shown high dislodgement rates in the atrium. For instance, another study looking at both pacemakers and biventricular devices also showed that the right atrial lead had the highest rate of dislodgement (1.9\%) followed by right ventricular (RV) implantable cardioverter-defibrillator lead (1.8\%), coronary sinus lead (1.4\%) and RV pacing lead (0.3\%) [5].

The reason for the consistently high rates of atrial lead dislodgement is unclear. Intuitively, it appears that it could be because the atrial lead is essentially free floating, with less support compared to RV apical leads which are wedged into a narrow space at the apex. It may also be that the extendable helix may not screw as well into the myocardium when in a J-curve versus a straight line.

Unfortunately, the present study does not delve into the details of implant parameters. Ghani et al. [5] noted in their study that lead dislodgements could have been prevented by adequate fixation of suture sleeves in one-third of cases. Also, the current of injury at implantation has been shown to predict adequate fixation of leads, but this was not reported in the present study [6].

\section{LEAD FAILURE}

This study finds that the independent predictors of lead failure include subclavian access, unipolar lead construction, younger patients, polyurethane $80 \mathrm{~A}$ insulation, and non-Biotronik leads. While some of these findings are consistent with prior studies [7], the manufacturer-based differences are a novel finding. These differences could be secondary to the polyurethane $80 \mathrm{~A}$ insulation that was used in certain older leads, but most of the modern leads use polyurethane 55D which is a more reliable material. As the authors suggest, these findings should be interpreted with caution until there is validation from larger multicentre studies.

This study has significant strengths. The data are from a single well-experienced centre which reduces bias related to interoperator variability. There are also a low number of patients lost to follow-up, a large number of leads, a very

\section{Address for correspondence:}

Jordan M. Prutkin, MD, MHS, Box 356422, 1959 NE Pacific Street, Seattle, WA 98195, USA, tel: (206) 685-4176, fax: (206) 616-1022, e-mail:jprutkin@uw.edu Kardiologia Polska Copyright (C) Polish Cardiac Society 2018

Note: The opinions expressed by the authors are not necessarily those of the journal editors, Polish Cardiac Society or publisher. 
large total patient-years of follow-up, and a variety of leads studied over this period.

There are some important methodologic limitations to note in the study. Lead failure was defined as an elevated pacing threshold or sensing problems. However, not every change in pacing threshold or sensing abnormality is due to the lead. Physiological states such as electrolyte imbalances, hypoxia, hypo/hyperglycaemia, myocardial ischaemia and infarction, cardiomyopathy, certain medications, and endocrine disorders may all cause changes in sensing or threshold which are not lead malfunctions [8].

The authors do not differentiate between various modes of lead failure such as lead fracture or insulation breaches. They also do not distinguish minor lead integrity issues which could be programmed around from catastrophic lead failure which would require a reoperation. For the patient and operator, this is probably the more important endpoint.

Lastly, this study only examined dual chamber systems and is not relevant to single chamber or biventricular systems which may have different lead-lead interactions or lead construction.

Therefore, there are several factors that underestimate or overestimate lead failure in this analysis.

Nevertheless, studies like this are helpful to inform patients about long-term risks, give insight about ways to improve lead placement by operators, instruct manufacturers about lead types and designs that are less effective in the long term, and provide post-market surveillance for regulatory agencies.

This study also emphasises the importance of continued development of leadless pacing systems, where there is no lead to fail. Only single chamber leadless pacemakers are currently available for routine use; and because of their novelty, we do not have long-term data for these devices. One-year follow-up of the Medtronic Micra and three-year follow-up of the Abbott Nanostim show promising stability of pacing and sensing parameters $[9,10]$, but there have been reports of premature battery failure with Nanostim [11] which will need to be corrected in later-generation devices.

In the near-term time horizon, we will likely use single chamber VDD and multicomponent dual chamber leadless pacemakers, as well as those which communicate with the subcutaneous implantable cardioverter-defibrillator [12]. Studies like this one from Dębski et al. [4] on transvenous pacemaker leads will hopefully be less needed. Leads continue to be the "weakest link" in the chain of cardiac pacing [13] but it appears that we are at the brink of the much-needed next revolution in cardiac pacing - a world without leads!

Conflict of interest: none declared

\section{References}

1. Lec SL. (Translated from Polish by Jacek Galazka). Unkempt Thoughts. Minerva Press, New York. 1967.

2. Aquilina O. A brief history of cardiac pacing. Images Paediatr Cardiol. 2006; 8(2): 17-81, indexed in Pubmed: 22368662.

3. Gillis AM. Lead Abandonment or Lead Extraction?: Weighing the Risks. JACC Clin Electrophysiol. 2017; 3(1): 10-11, doi: 10.1016/j. jacep.2016.09.005, indexed in Pubmed: 29759688.

4. Dębski M, Ulman M, Ząbek A, et al. Lead-related complications after DDD pacemaker implantation. Kardiol Pol. 2018; 76(8): 1224-1231, doi: 10.5603/KP.a2018.0089, indexed in Pubmed: 29633234.

5. Ghani A, Delnoy PP, Ramdat Misier AR, et al. Incidence of lead dislodgement, malfunction and perforation during the first year following device implantation. Neth Heart J. 2014; 22(6): 286-291, doi: 10.1007/s12471-014-0556-6, indexed in Pubmed: 24807834

6. Saxonhouse SJ, Conti JB, Curtis AB. Current of injury predicts adequate active lead fixation in permanent pacemaker/defibrillation leads. J Am Coll Cardiol. 2005; 45(3): 412-417, doi: 10.1016/j. jacc.2004.10.045, indexed in Pubmed: 15680721.

7. Aizawa Y, Negishi M, Kashimura S, et al. Predictive factors of lead failure in patients implanted with cardiac devices. Int J Cardiol. 2015; 199: 277-281, doi: 10.1016/j.ijcard.2015.07.055, indexed in Pubmed: 26218183.

8. Dohrmann ML, Goldschlager NF. Myocardial stimulation threshold in patients with cardiac pacemakers: effect of physiologic variables, pharmacologic agents, and lead electrodes. Cardiol Clin. 1985; 3(4): 527-537, indexed in Pubmed: 3910236.

9. Duray GZ, Ritter P, El-Chami M, et al. Long-term performance of a transcatheter pacing system: 12-Month results from the Micra Transcatheter Pacing Study. Heart Rhythm. 2017; 14(5): 702-709, doi: 10.1016/j.hrthm.2017.01.035, indexed in Pubmed: 28192207.

10. Tjong FVY, Knops RE, Neuzil P, et al. Midterm safety and performance of a leadless cardiac pacemaker. Circulation. 2018; 137(6): 633-635, doi: 10.1161/CIRCULATIONAHA.117.030106, indexed in Pubmed: 29431664.

11. Richter S, Döring M, Ebert M, et al. Battery malfunction of a leadless cardiac pacemaker. Circulation. 2018; 137(22): 2408-2410, doi: 10.1161/CIRCULATIONAHA.117.033371, indexed in Pubmed: 29540439.

12. Tjong FVY, Brouwer TF, Koop B, et al. Acute and 3-month performance of a communicating leadless antitachycardia pacemaker and subcutaneous implantable defibrillator. JACC Clin Electrophysiol. 2017; 3(13): 1487-1498, doi: 10.1016/j. jacep.2017.04.002, indexed in Pubmed: 29759829.

13. Hauser RG, Hayes DL, Kallinen LM, et al. Clinical experience with pacemaker pulse generators and transvenous leads: an 8-year prospective multicenter study. Heart Rhythm. 2007; 4(2): 154-160, doi: 10.1016/j.hrthm.2006.10.009, indexed in Pubmed: 17275749. 